\title{
PMS development in local public transport: Comparing Milan and Amsterdam
}

\author{
Deborah Agostino ${ }^{\mathrm{b}}$, Bauke Steenhuisen ${ }^{\mathrm{a}, *}$, Michela Arnaboldi ${ }^{\mathrm{b}}$, Hans de Bruijn \\ a \\ ${ }^{a}$ Faculty of Technology, Policy and Management, Jaffalaan 5, 2628 BX Delft, The Netherlands \\ ${ }^{\mathrm{b}}$ Politecnico di Milano, Dipartimento di Ingegneria Gestionale (DIG), via Lamburschini 4/b, Milan, Italy
}

Available online 13 March 2014

\section{Introduction}

European transportation reforms have considerably changed the way governments have dealt with the provision of the public transport from the 1990s onwards. Market mechanisms have been introduced through competitive tendering (Docherty et al., 2004). This movement is part of a wider appearance of private management styles in many other public sectors (Hood, 1991) with the principal aim to improve the quality of service and reduce associated costs.

Central to this movement, in relation to New Public Management (Hood, 1991), is the introduction of Performance Measurement Systems (PMSs), defined as 'the set of metrics used to quantify both the efficiency and effectiveness of actions' (Neely et al., 1995, p. 81). PMSs are a core mechanism in the modernisation of public services for setting targets and incentives, controlling private providers, externally accounting for performance, learning and continuously improving service provision (Behn, 2003; Johnsen, 2005).

PMSs have also been a cornerstone in the transportation reforming processes across Europe. Several studies have explored and discussed their merits in the past. These studies typically focus on technical aspects, such as the type of indicators (Hensher and Daniels, 1995; Badami and Haider, 2007; Nathanail, 2008; Chen et al., 2009; Börjesson and Eliasson, 2011; Mishra et al., 2012; Hassan et al., 2013), the use of targets and thresholds for setting incentives (Hidas and Black, 2002; Marsden and Bonsall, 2006; Marsden et al., 2009) and the type of information required in performance-based contracts (Hensher and Stanley, 2003). These studies display the heterogeneity of PMSs, but they do not address the process by which these PMSs and their heterogeneity develop.

This paper looks into the process of PMS development, defined as the trajectory from design to redesign and from the initial use of the PMS to new ways of use. PMS development is an essential phenomenon, as the use of PMS will always bring issues to light that can be optimised in the PMS design. Real-world dynamics in transportation sectors cannot be adequately captured in a PMS that is entirely static. PMS development has been evidenced in other sectors (e.g. accounting) to have a high impact on the ultimate design of the PMS, its use and actors' processes of learning, both about the PMS and the service delivery (Preston et al., 1992; Chua, 1995; Gendron et al., 2007; Arnaboldi and Azzone, 2010).

PMS development is a multi-actor process (de Bruijn and ten Heuvelhof, 2008), since multiple actors discuss and decide over time on the PMS content, its structure and uses. These actors for example are the governments using the PMS as an instrument (referred to as service regulator), the companies providing services subject to the PMS (referred to as service provider) and the users of services. Though a PMS design can theoretically be decided uni-laterally by a government, PMS use also depends on how companies provide information and respond to the PMS incentives. PMS development is shaped by this interaction between actors.

In this paper, we investigate how the PMS design and its use developed in two different cities in two different countries. Empirically, the research is based on two case studies, Milan and 
Table 1

Dimensions of PMS.

\begin{tabular}{ll}
\hline PMS design & KPIs, targets, and system of reports \\
PMS use & Type of use, data validation, data interpretation and effects \\
PMS development & Changes in PMS design and changes in PMS use \\
\hline
\end{tabular}

Amsterdam. These two European cities reformed their transportation sectors at more or less the same time. Trigger for the reforms was the enactment of European Union rules in 1991 to liberalise public transport. During the reforming process in both cases, there was never any discussion as to whether PMS was a useful instrument. Yet the modes through which the PMS was adopted, implemented, used and evolved were different in the two cities.

Results show a similar PMS design but a diverse use and surprising path of development, in particular with regard to the involvement of actors. The national culture, made visible in the governance structure, is used as explanatory lens to interpret the differences in PMS development.

\section{PMS: linking design, use and development}

The investigation of PMS development is grounded in the accounting literature (e.g. Preston et al., 1992; Chua, 1995; Gendron et al., 2007; Arnaboldi and Azzone, 2010) and starts from the assumption that PMS is shaped through three elements: design, use and development (Table 1). The first element of analysis is the PMS design, also referred to as the technical characteristics of PMS. Its key components are KPIs, targets with the associated system of bonuses and penalties, and a system of reporting (Otley, 1999).

Second element of analysis is PMS use, which involves the investigation of practices of interpreting and validating data and the real-world effects associated with PMS use. Data validation considers how the validity of the provided performance information is checked and by whom. PMS effects refer to the penalties, bonuses and other effects, both intended and perverse, triggered by the use of performance measures.

Third element of investigation is PMS development. This includes how actors, their mutual relationships, their competences and their involvement lead to evolutionary and other changes in PMS design and use.

Differences and similarities in PMS development can be explained considering the institutional settings, namely the national culture and the governance embedded into this culture (e.g. de Waal, 2005). A common dichotomy in literature on regulation styles (Hawkins 1983; Sparrow, 2000) is 'hierarchy' versus 'dialogue'. This dichotomy considerably overlaps with the dichotomy of 'masculine' and 'feminine' cultures (Hofstede, 1984). In our two case studies, according to Hofstede (1984), Italy has a masculine culture and the Dutch culture is relatively feminine. We further refine and elaborate on this explanatory lens when discussing the results below.

\section{Research approach}

In order to investigate the PMS development process, we adopted a multi-country case study (Yin, 1994). It is based on a longitudinal comparison, i.e. comparing cases over a time horizon of 15 years during the transportation reforming process of two European cities: Milan and Amsterdam. The case study methodology was selected because it is considered the most useful approach for understanding complex processes and the most appropriate for conducting exploratory investigations (Yin, 1993). It gave us the possibility of entering in the details of PMS practice, its technical characteristics, its uses and its evolution over time.

\subsection{Data collection, analysis and presentation}

Data have been collected longitudinally from a wide variety of sources, including various documents, formal semi-structured interviews, informal conversations and site visits. Particularly semi-structured interviews allow focusing on the specific research interests, to open up the real-world context of PMS (Weiss, 1995).

Several documents have been analysed, such as laws, official reports, internal reports, media articles and scientific studies directly relevant to the cases (e.g. Veeneman, 2010). Legislative decrees, national laws and regional laws on public transport have been collected and analysed to understand the legislative reference context. Official reports include annual financial reports and mobility charts published periodically on the website of the transportation companies in Milan and Amsterdam. This data source provided insights to explore PMS design in terms of KPIs adopted. Internal reports relate to documents on PMS internally developed by the service providers for the regulators and not available to users. These reports showed evidence on PMS design with specific reference to KPIs and targets.

For each case, interviews were carried out with local government authorities and managers of the service providers. In total, we conducted 31 interviews during the period 2009-2012. Interviews lasted on average $1 \mathrm{~h}$, and were transcribed and textually analysed in order to identify any interesting pattern among the interviews. A summary was then sent to each interviewee for further clarifications and the final approval. Additionally, site visits in local administrations, offices of the transportation companies and of users' representatives proved insightful. Informal conversations also were particularly useful to gain additional information on PMS design and use and validate insights from formal interviews.

The Amsterdam results were based on two interviews, but the case relied on a wider set of empirical data, which included multiple official and internal reports and previous research (Veeneman, 2010). The choice of the empirical strategy was due to large availability and accessibility of documents on the investigated issue. Furthermore, for the Amsterdam case, an extra interview was carried out with an expert to reflect on the validity and completeness of previous collected material. The Milan case required a different strategy. The reports available for the researchers were not sufficient to investigate the phenomenon. Moreover, the reconstruction of the PMS design and implementation required to interview many actors, each of them having a partial vision on the system.

\subsection{Introducing the cases: Milan and Amsterdam}

Milan is the second largest municipality in Italy and the capital of the Lombardy Region. The city represents an important transport node for the country, being one of the most important hubs for air, rail, and road networks, and local public transport. There are 4,757,000 travellers every day (Agenzia Milanese Mobilità e Ambiente, 2006), of whom 53\% travel entirely within the municipality, with the remaining $47 \%$ entering and exiting the city.

The local public transport serves an area of $703 \mathrm{~km}^{2}$ with 2,754,258 inhabitants (see Table 2). The urban transport network consists of 97 bus routes, 19 tram routes, 3 metro routes and 3 trolley bus routes providing the service both inside the city and between the city and the nearby 51 provincial towns.

ATM, a public limited company owned by the municipality, is the service provider. The company was founded in 1931, when it 
started the local service provision in the municipal area. Its activities then expanded to the management of car parks, car sharing and bike sharing, and outside the city with the funicular railway in Como, the automated underground in Copenhagen and, since 2011, the metro service in Riyadh. ATM won the bid issued by the Municipality of Milan, which represents the service regulator. The activities of both regulators and providers are defined by the Lombardy Region in general guidelines. Specifically, the Region sets guidelines for service provision in the local area. The municipality is in charge of implementing these guideline through a contract signed with the transportation company that delivers the service. The structure of the local public transport system is graphically represented in Fig. 1.

Amsterdam is the largest city and the capital of the Netherlands. The entire metropolitan area around Amsterdam has an urban population of around 1.4 million inhabitants. Local public transport is constituted by a network of 34 bus routes, 16 tram routes, 4 metro routes and 5 ferry routes. The local service covers an area of $1815 \mathrm{~km}^{2}$, twice the area of Milan, but the number of inhabitants in the area is lower (see Table 2), as is the number of transported passengers, who numbered around 204 million in 2011.

GVB is the service provider, wholly owned by the municipality of Amsterdam. The company was established in 1900 for providing the bus and tram service, and it has included the ferry services since 1943. GVB signed its first service contract in 2006 and a new one in 2012. In the Amsterdam case, transport guidelines are enacted by the National government. The city region (not the municipality) is the service regulator. This is different from the

Table 2

Comparison between ATM and GVB in 2011.

\begin{tabular}{lll}
\hline & ATM & GVB \\
\hline Transported passengers & 682 million & 204 million \\
Served area (in $\mathrm{km}^{2}$ ) & 703 & 1815 \\
Inhabitants in the served area & 2.7 million & 1 million \\
Bus fleet & 1334 & 211 \\
Tram fleet & 553 & 216 \\
Metro fleet & 939 & 104 \\
Boat fleet & 0 & 12 \\
Trolleybus fleet & 149 & 0 \\
Total revenues (in $€$ ) & 502 million & 469 million \\
Operating costs (in $€$ ) & 513 million & 421 million \\
Net income (in $€)$ & 4 million & 36 million \\
\hline
\end{tabular}

Italian context, where guidelines are set at a local level by the Region and the Municipality plays the role of service regulator.

A comparison table summarises the transport systems in the two cities (Table 2).

\section{Results}

This section presents PMS design, use and development per each case separately. Next, the comparative section discusses similarities and differences in PMS between the two cities and explores the role of the national culture in shaping the divergence in PMS.

\subsection{Milan}

\subsubsection{PMS design}

The PMS was introduced with the Regional Law 22/98 as a control mechanism to regulate the relationship between the service provider and the service regulator. The service contract is the document that specifies PMS technical features in terms of KPIs, targets and associated reporting documents.

4.1.1.1. Technical characteristics. The type of measures that the service provider (ATM) has to report to the service regulator is described in the service contract. These measures consist of 40 KPIs, mainly related to service effectiveness. Indicators include service regularity, service punctuality, comfort, cleanliness, accessibility of the transport modes, environment, safety, the quality of travel information provided to users and customer satisfaction. These data are further complemented with other KPIs related to the quantity of the service and its costs: number of transported passengers, passenger per kilometre, saturation of buses, production costs, personnel costs and revenues.

Targets are set by the service regulator. They are not negotiated with the service provider. For each KPI in the service contract, a threshold level is specified every year. A system of bonuses and penalties is linked to achieving or not these threshold levels.

Reporting is also regulated in the service contract. Two types of documents are required. First, the mobility chart, delivered every year by the service provider to citizens. It is a leaflet of 20 pages that summarises the activities of the company during the year and also includes some performance measures. Specifically, the document starts with a description of the company and the main event or changes that characterised the company or the service provided
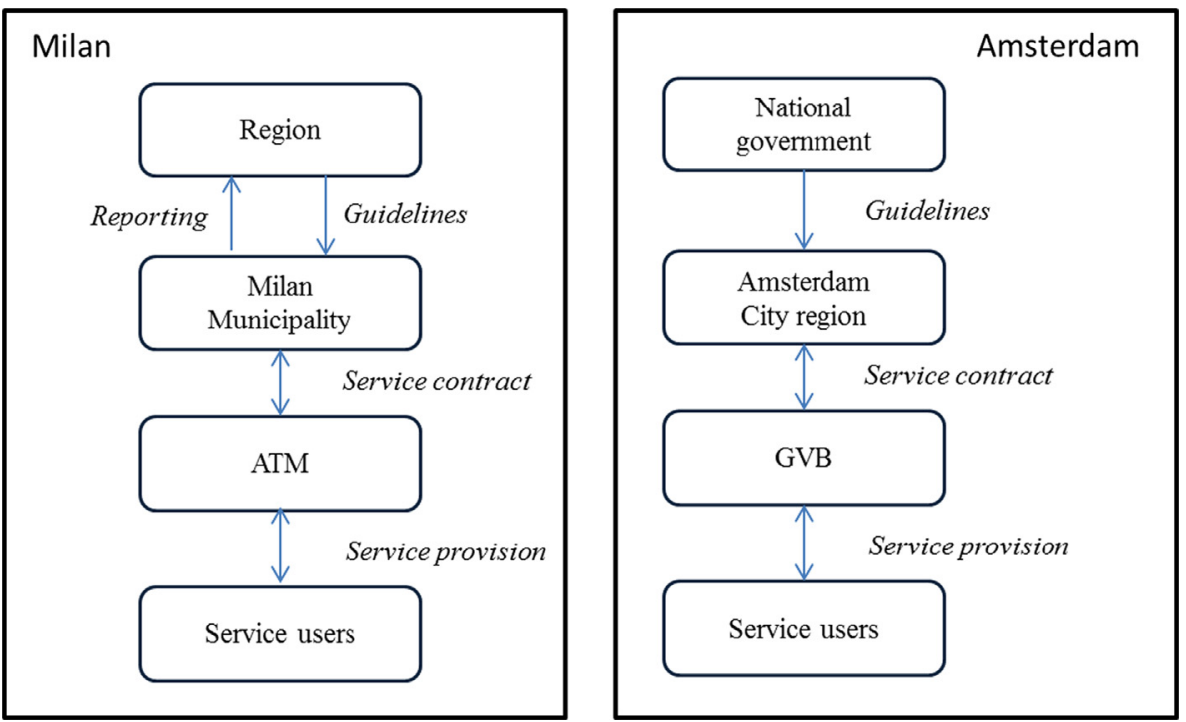

Fig. 1. Structure of the local public transport system in Milan and Amsterdam. 
over the year. After this introduction, a detailed analysis of performance measures is provided. These data, defined in the service contract between the company and the Municipality, refer to customer satisfaction, punctuality, regularity, accessibility, comfort and cleanliness. Second, ATM also provides reports to the Municipality of Milan. These consist of annual reports and three mid-year reports. These reports include not only measures about the service quality, but also information about costs of the service and quantity of the service provided.

\subsubsection{PMS use}

The type of PMS use was also regulated with the Regional Law $22 / 98$ that defined the way in which actors had to engage with performance measures. The Region adopted a hierarchical type of governance. This approach was acknowledged by the Region as the most suitable approach for ensuring a strict control over service performance: measures and targets were assigned by the municipality to ATM at the end of the quarter and of the year, reports were collected, performance was compared to the threshold levels and the relative bonuses were assigned. Both the service regulator and the service provider maintained a narrow focus on compliance with formal targets.

Besides this formal description, the PMS was in practice adopted by the actors in the network in different ways: the municipality of Milan and ATM used the PMS to comply with the regulation, while for the Region and users of the system it represented a tool for understanding and interpreting the transportation context.

4.1.2.1. Data validation and interpretation. Neither the Municipality nor ATM is actively committed to validating or interpreting data. According to the administrator, represented by the Lombardy Region, the municipality has a 'paper pusher role' (in the words of an informant), simply transmitting reports to the Region. According to a representative of the users, "the Municipality has never checked numbers included in the report and it is not in charge of verifying whether the information provided by ATM are [sic] trustable or not'. ATM uses PMS only to the extent necessary to comply with the regulation. The reason behind this lies in the technical features of PMS design and the way in which the KPIs have been selected, which are recognised as being 'too generic to support internal decision making', as articulated by a controller at ATM. By contrast, the Municipality of Milan, which had never adopted performance measures to manage service provision before the introduction of competitive tendering procedures, considers the PMS a big innovation.

4.1.2.2. PMS effects. The PMS in Milan involves targets for all KPIs defined in the contract. Usually, these targets are far lower than the status quo, generating no direct incentives from the PMS.

Although there were no direct incentives, the PMS triggered indirect learning effects beyond the contract relationship. The Region and user associations tried to use the PMS, interpreting data and learning lessons. User associations used the PMS to develop their technical competences over the years with the purpose to objectively describe the transport system and suggest solutions for improving the service. The Region also used the PMS to develop knowledge about the regional network, highlighting problems, proposing solutions and further refining in the reform. For example, data about customer satisfaction and future financial projections on EBITDA were key figures in prompting the Region to reorganise the network system.

\subsubsection{PMS development}

While PMS design has remained stable over time, the PMS use has evolved in particular with the involvement of user associations.
Though both the service regulator and the service provider maintained their focus on strict compliance with formal targets, a development in the PMS use occurred outside the contract relationship.

Users, particularly the organised association of users, have always been interested in the performance of local public transport, but they were neither involved in the reforming processes, nor in the formal activities of PMS design and use. Through associations, users collected information about how ATM was performing and shared their results on the website or during ad hoc informal meetings. The main reason behind this spontaneous process of informal data collection and external sharing lies in the distrust in the data provided by service providers. According to representatives of users, data included in mobility charters are manipulated to give the impression that the results are better than they are. Over time, the representatives of users became able to contest the available formal data with their own data.

Due to this active approach towards PMS, users developed competences in PMS over time. Because of these competences, they have been invited to regional meetings since 2007 to discuss the new service network configuration. Representatives of users, who had always played a marginal and informal role, were gradually granted more influence because of their recognised competences in transportation issues. In March 2012, the new regulation approved the formal recognition of the role of users in using PMS.

\subsection{Amsterdam}

This case description is similarly structured in the three elements: PMS design, PMS use and PMS development.

\subsubsection{PMS design}

The goal of PMS in the Amsterdam case is not strictly about compliance with formal targets. In 2012, the tender formulated its goal as 'continuous improvement at a good price to realise the ambitions of the City region'. Nevertheless, the PMS design focuses mainly on compliance with 'the ambitions'.

4.2.1.1. Technical characteristics. Amsterdam has a range of KPIs concerning capacity (planned and realized), punctuality, passenger numbers, accessibility for people with disabilities, social safety and quality of customer service. These KPIs and their targets are defined by the transport regulator in the service contract. There are bonuses for reaching targets and penalties for failing to do so. There is a differentiation per target. Amsterdam focuses on departure punctuality and cancellations. The indicators on cancellations and punctuality are explicitly prioritised over others in determining the relative bonuses and penalties.

GVB is not directly accountable for its yearly performance to passengers. Amsterdam has no mobility charter for the transport company to directly inform the passengers on past performance and future targets. However, GVB is accountable for its performance to the transport regulator. Quarterly reports are drawn up in relation to the service contract. These reports are not public but considered confidential.

\subsubsection{PMS use}

Compliance with targets is also a central theme, but, at the same time, interaction and dialogue between the city region and the transportation company is facilitated by the PMS in. This interaction is focused on 'continuous improvement' and data interpretation.

4.2.2.1. Data interpretation and validation. In Amsterdam, the contract relationship between the transport regulator and GVB is 
central when interpreting the PMS. Dialogue between the city region and GVB serves to find the reasons for underperformance, to prioritise improvement measures and to fine tune additional or more specific demands.

The transport authority also actively checks and validates the GVB reports. The city region has a large variety of mechanisms for checking the data provided by the company. These include measuring a reliability criterion for the reported data, looking into a real-time database, generating information by means of an electronic ticketing system, doing spot checks, using mystery guests and hidden cameras or hiring an independent consultancy to study technical aspects or customer satisfaction.

4.2.2.2. PMS effects. In 2006, GVB incurred a series of penalties. In 2011, it earned the maximum bonus of 2.5 million euros, $7 \%$ of its annual profits. In these 6 years, the PMS has reported an improved performance across all indicators. For example, the KPI for punctuality was about $70 \%$ in 2006 , rising to $94 \%$ in 2012 . The incentives and the regular discussions with the city region actively focused on this improvement.

A range of other learning effects can be mentioned. In a more indirect way, for example, the attitude towards complaints by customers changed, as observed by the respondents. As the dialogue about the PMS developed, the company became more interested in the information provided by these complaints as a means of improving performance. The dialogue between the service regulator and the service provider on the PMS also identified a variety of bottlenecks and solutions. These included measuring customer satisfaction differently, adding requirements to invest in real-time travel information in trams and at stops, formulating a new rule that at least every other bus/tram should have a low floor. Incidentally, the city region also adjusts targets and adds or drops targets.

\subsubsection{PMS development}

The PMS design underwent some moderate changes with regard to the set of targets, the exact articulation of indicators and how certain indicators are measured, in particular customer satisfaction. These incidental changes refined the PMS but did not drastically change its scope, ambition or functioning.

The PMS use evolved over time. At first, the new managerial relationship between the service provider and the service regulator led to conflicts. In the first 2 years, eight lawsuits were planned but eventually settled with the help of mediation. Over time, the service provider and the service regulator found their way in using the PMS as a basis for assigning penalties and bonuses as well as for dialogue on how to improve the service.
The involvement of the main platform for customer associations has been low ever since the PMS design was implemented. The customer associations were involved in the design phase but not in the use of PMS.

The national government kept aloof from PMS design and use, but nevertheless had a major influence on the new contract in 2012. This started as a contract up to 2018 with an agreed investment package of 120 million euros. After the contract had been signed, the new national government enforced severe budget cuts and made competitive tendering compulsory. This forced the Amsterdam city region to drastically change the contract. Its duration was halved and the required investments reduced by a third, with corresponding repercussions for the relevant targets.

\subsection{Comparison}

The PMS characteristics for Milan and Amsterdam are summarised and compared in Table 3.

The technical design of PMS was generally similar in the two cities while the PMS use and development differ in the two cases.

As far as the PMS use is concerned, the main difference concerns data interpretation from PMS. In Milan the interpretation of data is a 'monologue' by the service regulator who decides whether the performance has met the required standard or not. Also in Amsterdam the PMS use focuses on compliance with targets. The difference lies in the dialogue between the service regulator and the service provider, supported by the PMS that is considered of comparable importance. As far as the PMS development is concerned, there are changes in PMS design and use, mainly attributable to actors involvement. The Dutch have explicitly organised the involvement of user associations in the PMS design phase. This nevertheless did not result in very active involvement of the user association in PMS development. In Milan user associations were not involved in the phases of design and use, but in the course of time, users got a powerful position because they prove to be very knowledgeable, thanks to the data provided by the PMS. It is paradoxical: the closed process in Milan indirectly caused an increased involvement of the user association, whereas the open process in Amsterdam led to a relatively closed contract relationship. Consequently, learning in the Amsterdam case is internally driven, whereas in Milan it is externally driven. Put differently, in both cases there is input from customer associations as checks and balances in the process of PMS development. In the Amsterdam case, the checks and balances are organised 'upstream', during PMS design, and dry up downstream, during PMS use and development. In the Milan case, no checks

Table 3

PMS comparison.

\begin{tabular}{|c|c|c|c|}
\hline & & Milan & Amsterdam \\
\hline \multirow[t]{3}{*}{ PMS design } & KPIs & $\begin{array}{l}\text { Drawn up by service regulator with focus on service } \\
\text { quantity and quality }\end{array}$ & $\begin{array}{l}\text { Drawn up by service regulator with focus on service } \\
\text { quantity and quality }\end{array}$ \\
\hline & Targets & Set by service regulator including bonus and penalties & Set by service regulator including bonus and penalties \\
\hline & Reporting & $\begin{array}{l}\text { Periodic reports for service regulator and mobility charts } \\
\text { for customers }\end{array}$ & Periodic reports for service regulator \\
\hline \multirow[t]{6}{*}{ PMS use } & Type of use & Hierarchical governance & Interactive governance \\
\hline & & Compliance-oriented & Improvement-oriented \\
\hline & Interpretation & No interpretation of the PMS data & $\begin{array}{l}\text { Interactive interpretation of PMS data in discussions } \\
\text { between service regulator and service provider }\end{array}$ \\
\hline & Validation & No validation of the PMS data & Multiple ways to validate PMS data \\
\hline & Effects & No penalties or bonuses & Many penalties and bonuses \\
\hline & & Learning effects outside contract relationship & Learning effects within the contract relationship \\
\hline \multirow[t]{2}{*}{ PMS development } & Changes in PMS design & No development & Moderate, incidental \\
\hline & Changes in PMS use & Shift towards more dialogue with customer associations & Shift towards a more stable contract relationship \\
\hline
\end{tabular}


Table 4

Framework of PMS development issues.

\begin{tabular}{ll}
\hline PMS design & Hierarchical versus interactive \\
& Duty versus goal oriented \\
PMS use & Monologue versus dialogue \\
& Closed versus open \\
& Single versus multiple purpose \\
PMS development & Internally versus externally driven \\
& Upstream versus downstream checks and balances \\
\hline
\end{tabular}

and balances are organised upstream but spontaneously emerge downstream.

Characteristics of the national culture provide a possible explanatory variable for the different PMS development. Italy is recognised as a masculine country, in which a hierarchical approach to relationships and top-down impositions prevail. The Netherlands has a more feminine culture, in which interpersonal relationships and dialogue prevail over the hierarchical positions. These differences in the culture become visible when considering the process of governance in the two countries. The feminine and horizontal Dutch culture is translated into a horizontal way of cooperation, based upon the idea of co-governance (Bode, 2006). On the contrary, the masculine Italian culture becomes visible in a strong emphasis on hierarchy. As said, there is a strong misfit between hierarchy on the one hand and the reality of an interdependent, multi-actor world on the other hand. This tension usually results in the emergence of horizontal processes behind the hierarchical façade.

This distance is the main difference between Italy and the Netherlands. In the Netherlands, horizontal decision-making sets the tone, both at the public stage and the backstage. There are only minor differences between the two stages. In Italy, there are major differences between the public stage (hierarchy) and the backstage (horizontal coordination) or, put differently, there is a big gap between public stage and backstage.

We assert that the smaller or larger gap between the public stage and the backstage, which translates the main features of the feminine and masculine culture, explains a lot of the development of PMS. In the Netherlands, with its minor differences between public stage and backstage, a dialogue based system is quite a natural thing. From the initial phases of the reform, dialogue was part of the PMS. In Italy, with its large distance between the formal and real world, PMS started as a hierarchical system. However, behind that formal hierarchical system, a more dialogue-based system developed. A key actor, user organisations, managed to enter the system and rebuild it to a more dialogue based system. Gradually, the original hierarchical system transformed into a more dialogue based system.

\section{Conclusion}

EU market reforms assigned local governments the role of service regulator to design and use Performance Measurement Systems (PMSs) for local public transport. We studied this type of performance measurements for Milan in Italy and Amsterdam in the Netherlands. We studied the design, use and development of PMS over the years. PMS development has provided an essential complementary perspective when studying PMS design and use. Four more specific conclusions are derived.

First, both countries have a multi-layer, multi-actor governance structure. In this fragmented structure, we found a top-down approach in Milan, aimed at maximising formal compliance. In Amsterdam, the PMS was focused on dialogue and learning. The Amsterdam approach was embedded in the Dutch feminine culture with a strong tradition of horizontal governance. The Milan approach was embedded in the more hierarchical, masculine Italian culture.

Second, despite the top down approach, we also found learning processes in the Italian case. Thanks to the information that the PMS made available, user organisations managed to strengthen their position by using this information. Subsequently, they were involved in the process of use, which made this process much more horizontal than it initially was. Evidently, a top-down approach can arouse actors who were initially kept out of the process, a mechanism that can be found in literature (de Bruijn and ten Heuvelhof, 2008, pp. 119-120; Koffijberg et al., 2012).

The Dutch case shows the opposite. User associations were offered a formal role in PMS design from the beginning and committed themselves to the PMS in advance. Because of this early commitment, their involvement in the phase of use was much more limited, as there was less need for user associations to be involved in this phase.

Third, the empirical focus on PMS development resulted in a conceptual refinement of the 'top-down versus bottom-up/dialogue' dichotomy, commonly used in literature on regulation styles (Hawkins, 1983; Sparrow, 2000). Inductively, we reached a rich framework listing seven issues based on this central dichotomy. This framework is summarised in Table 4 below. Its core message is that the opportunities for dialogue are spread out over all three PMS elements: design, use and development.

Fourth, we found that PMS can be designed either with a focus on learning or with a focus on compliance. Our process perspective has unveiled the development and dynamics of a PMS, which eventually results in a PMS-in-action that differs substantially from the PMS-on-paper. It results in a much more balanced assessment of PMS's impact. It makes clear that there is hope for masculine countries with an inbuilt resistance to adopting a learning oriented approach. The main lesson of our research seems to be: PMS follows structure. In a multi-layer, multi-actor structured world, this structure will force the PMS to develop into a more horizontal, dialogue-based system.

\section{References}

Agenzia Milanese Mobilità e Ambiente, 2006. Studio per l'introduzione del road pricing a Milano. Available at $\langle$ www.amat-mi.it/it/documenti/tutti〉 (accessed 20.07.13)

Arnaboldi, M., Azzone, G., 2010. Constructing performance measurement in the public sector. Crit. Perspect. Account. 21 (4), 266-282.

Badami, M.G., Haider, M., 2007. An analysis of public bus transit performance in Indian cities. Transp. Res. Part A Policy Pract. 41 (10), 961-981.

Behn, R., 2003. Why measure performance? Different purposes require different measures. Public Adm. Rev. 63 (5), 586-606.

Bode, I., 2006. Co-governance within networks and the non-profit-for-profit divide. A cross-cultural perspective on the evolution of domiciliary elderly care. Public Manag. Rev. 8 (4), 551-566.

Börjesson, M., Eliasson, J., 2011. On the use of 'average delay' as a measure of train reliability. Transp. Res. Part A Policy Pract. 45 (3), 171-184.

de Bruijn, H., ten Heuvelhof, E., 2008. Management in Networks. On Multi-actor Decision Making. Routledge, New York.

Chen, X., Yu, L., Zhang, Y., Guo, J., 2009. Analyzing urban bus service reliability at the stop, route, and network levels. Transp. Res. Part A Policy Pract. 43 (8), 722-734.

Chua, W.F., 1995. Experts, networks and inscriptions in the fabrication of accounting images: a story of the representation of three public hospitals. Account. Organ. Soc. 20 (2/3), 111-145.

Docherty, I., Shaw, J., Gather, M., 2004. State intervention in contemporary transport. J. Transp. Geogr. 12 (4), 257-264.

Gendron, Y., Cooper, D.J., Townley, B., 2007. The construction of auditing expertise in measuring government performance. Account. Organ. Soc. 32 (1/2), 101-129.

Hassan, M.N., Hawas, Y.E., Ahmed, K., 2013. A multi-dimensional framework for evaluating the transit service performance. Transp. Res. Part A Policy Pract. 50, 47-61.

Hawkins, K., 1983. Bargain and bluff: compliance strategy and deterrence in the enforcement of regulation. Law Policy Q. 5 (1), 35-73.

Hensher, D.A., Daniels, R., 1995. Productivity measurement in the urban bus sector. Transp. Policy 2 (3), 179-194. 
Hensher, D.A., Stanley, J., 2003. Performance-based quality contracts in bus service provision. Transp. Res. Part A Policy Pract. 37 (6), 519-538.

Hidas, P., Black, J., 2002. Targets and performance indicators for sustainable

urbanansport: a review of current practice in Sydney, Australia. In: Brebbia, C.A. Martin-Duque, J., Wadhwa, L. (Eds.), The Sustainable City II. Urban Regeneration and Sustainability. WIT Press, Hampshire

Hofstede, G., 1984. Cultural dimensions in management and planning. Asian Pac. J. Manag. 1 (2), 81-99.

Hood, C., 1991. A public management for all seasons? Public Adm. 69 (1), 3-19.

Johnsen, J., 2005. What does 25 years of experience tell us about the state of performance measurement in public policy and management? Public Money

Manag. 25 (1), 9-17.

Koffijberg, J., de Bruijn, H., Priemus, H., 2012. Combining hierarchical and network strategies: successful changes in Dutch social housing. Public Adm. 90 (1), 262-275.

Marsden, G., Bonsall, P., 2006. Performance targets in transport policy.

Transp. Policy 13 (3), 191-203.

Marsden, G., Kelly, C., Nellthorp, J., 2009. The likely impacts of target setting and performance rewards in local transport. Transp. Policy 16 (2), 59-67.

Mishra, S., Welch, T.F., Jha, M.K., 2012. Performance indicators for public

transit connectivity in multi-modal transportation networks. Transp. Res. Part A Policy Pract. 46 (7), 1066-1085.
Nathanail, E., 2008. Measuring the quality of service for passengers on the hellenic railways. Transp. Res. Part A Policy Pract. 42 (1), 48-66.

Neely, A., Gregory, M., Platts, K., 1995. Performance measurement system design. A literature review and research agenda. Int. J. Oper. Prod. Manag. 15 (4), 80-116.

Otley, D., 1999. Performance management: a framework for management contro system research. Manag. Account. Res. 10 (4), 363-382.

Preston, A.M., Cooper, D.J., Coombs, R., 1992. Fabricating budgets: a study of the production of management budgeting in the National Health Service. Account. Organ. Soc. 17 (6), 561-593.

Sparrow, M., 2000. The Regulatory Craft. Brookings Institution Press, Washington DC.

Veeneman, W., 2010. Changing public transport governance in Dutch metropoles: to tender or not to tender. Res. Transp. Econ. 29 (1), 195-203.

de Waal, A., 2005. The role of behavioral factors and national cultures in creating effective performance management systems. Syst. Pract. Action Res. 19 (1), 61-80.

Weiss, R.S., 1995. Learning From Strangers. Free Press, New York

Yin, R.K., 1993. Application of Case Study Research, 2nd ed. Sage, Thousand Oaks, CA

Yin, R.K., 1994. Case Study Research: Design and Methods, 2nd ed. Sage, Thousand Oaks, CA 